\title{
WEITERE ZEUGNISSE UBER JOHANN VON MORSHEIM.
}

Bei Goedeke im Grundriss 12,392 ist zusammengestellt, was wir von urkundlichen zeugnissen ther Johann von Morsheim kennen und Beitr. 12, 504 hat Sievers noch ein weiteres hinzugefugt. Im folgenden sollen mehrere nachweise mitgeteilt werden, wie sie eine neue urkundenpublication, der 3. teil der Quellen zur gesehichte der stadt Worms bietet. Ich bemerke noch, dass nach einer gutigst durch die grossherzogliche burgermeisterei Worms vermittelten äusserung des städtischen archivars, herrn prof. dr. Weckerling, das dortige archiv, soweit bekannt, kein weiteres material in bezug auf Johann von Morsheim enthält.

Ich lasse die belege in chronologischer reihe folgen:

1487 (nov.) wird Hans von Morszheym fauit z⿺辶 Germerszheym unter den räten der Pfalz bei einem streite der stadt Worms mit ihrem bischof genannt (Boos 3, 550, 28).

1487 (nov. 29) in gleicher angelegenheit: Hans von Morszheym fait zu Germersheym ibid. 3,551, $21 \mathrm{f}$.

1487 (nov. 30) vertritt Hans von Morszheym in einer längeren rede die meinung der pfalzgräflichen räte.

Zu ende des jahres 1491 wird er zuletzt als vogt von Germersheim erwähnt (Goedeke 12,392). Er ist dann burggraf von Alzei geworden. Wir können ihn als solchen zuerst am 3. october 1494 nachweisen, wo er wider unter den pfälzischen räten in die Wormser händel eingreift. Er wird aufyefuhrt als Johann Morschemer, der zit burggraf zu Altzey (Boos 3, 383, 22). In streitereien zwischen Worms und einigen dörfern der umgegend uber den viehtrieb im Waag nahm er mit einigen amtleuten und Wormser ratsangehörigen einen augenschein ab, und bei dieser gelegenheit teilen die Acta Wormatiensia 1 fol. 40 ein dictum Hansens von Morsheim mit, das Boos (3,384, 38 ff.) erwähnt und das auch ich, da es die persönlichkeit des mannes berthbrt, mitteilen will: Als man nu wider uber den 
viehe wegk zur statt zu ritte, ritten die amptleute nebeneinander und sahen die stadt an und sonderlich das newe bollwerck, das mun in demselben 94. jare angefangen und auffgebawet hett hinder Nonnenmonster am ort; sagt der burggrave Hans Morszheimer, man hett da herausz gebawet auff des bischoffs gerechtikeit, dann man gestunde dem rat nit neiter dann als ferr die mawr begriff und nit einen schuch herausz, das must man wider abthun. Auch sagt der burggrave zu den andern hupschlich, doch das es die ratsfrunde horten, als er das monster ansahe: es ist ymmer schade, das der stifft vergeen soll. Sagt der bürger einer, soll er dann vergeen? Sagt er, ja, es mag darzu komen, das man pferd darinn stellen wirdet. Die und dergleichen speyhewortte wurden viel geredt etc.

Am 11. nov. 1494 hält Johann von Morsheim dann das von Sievers a. a. 0. erwähnte gericht zu Heppenheim. Am 21. juli 1498 wird in einer Wormser urkunde (Boos 3, 575,4) ein burggraf ron Altzey ohne weitere bezeichnung erwähnt, aber grade dieses fehlen näherer angaben legt uns nahe, auch hier an Johann von Morsheim zu denken. Im ersten jahre des neuen jahrhunderts, am 10. august 1501, tritt dann Hans Landschad als burggraf von Alzei auf (Boos 3, 446, 28) und am 29. januar 1502 wird Hans von Morszheim ohne weiteren zusatz eines amtes unter den pfälzischen răten genannt; hofmeister ist er damals noch nicht (Boos 3, 470, 32 und 49).

Der von Goedeke (a. a. o.) erörterte lehnsrevers des Hans Melchior von Morsheim an den pfalzgraf bei Rhein vom 5. märz 1532 würde, falls dieser ein sohn Johanns von Morsheim gewesen wäre, vermuten lassen, dass der dichter erst kurz vorher, anfang der dreissiger jahre, gestorben sei. Unter dieser voraussetzung wlirden wir in dem pfälzischen hofmeister, der im april des jahres 1526 die endguiltige beilegung der kämpfe zwischen stadt und pfaffheit von Worms herbeifuhrt, Johann von Morsheim sehen dürfen. Aber diese annahme bleibt immerhin unsicher, und ich wage nicht, darauf fussend, die in einer längeren rede (Boos 3,634, $4 \mathrm{ff}$.) niedergelegten anschauungen des hofmeisters tuber die zeitverhältnisse zur charakterzeichnung Johanns von Morsheim zu verwerten.

HALLE A. S., januar 1894.

JOHN MEIER. 